\title{
« Fin de vie » et renaissance clandestine en Chine du Sud
}

Quand des « déchets » redeviennent des écrans plats

"End of Life" and Clandestine Rebirth in South China. On "Waste" that

Transforms Back into Flat Screens

\section{Yvan Schulz}

\section{OpenEdition}

\section{Journals}

Édition électronique

URL : https://journals.openedition.org/tc/7856

DOI : $10.4000 /$ tc. 7856

ISSN : 1952-420X

Éditeur

Éditions de l'EHESS

Édition imprimée

Date de publication : 31 octobre 2016

Pagination : 158-161

ISBN : 9782713225291

ISSN : 0248-6016

Référence électronique

Yvan Schulz, « «Fin de vie » et renaissance clandestine en Chine du Sud », Techniques \& Culture [En ligne], 65-66 | 2016, mis en ligne le 31 octobre 2016, consulté le 29 septembre 2022. URL : http:// journals.openedition.org/tc/7856; DOI : https://doi.org/10.4000/tc.7856 


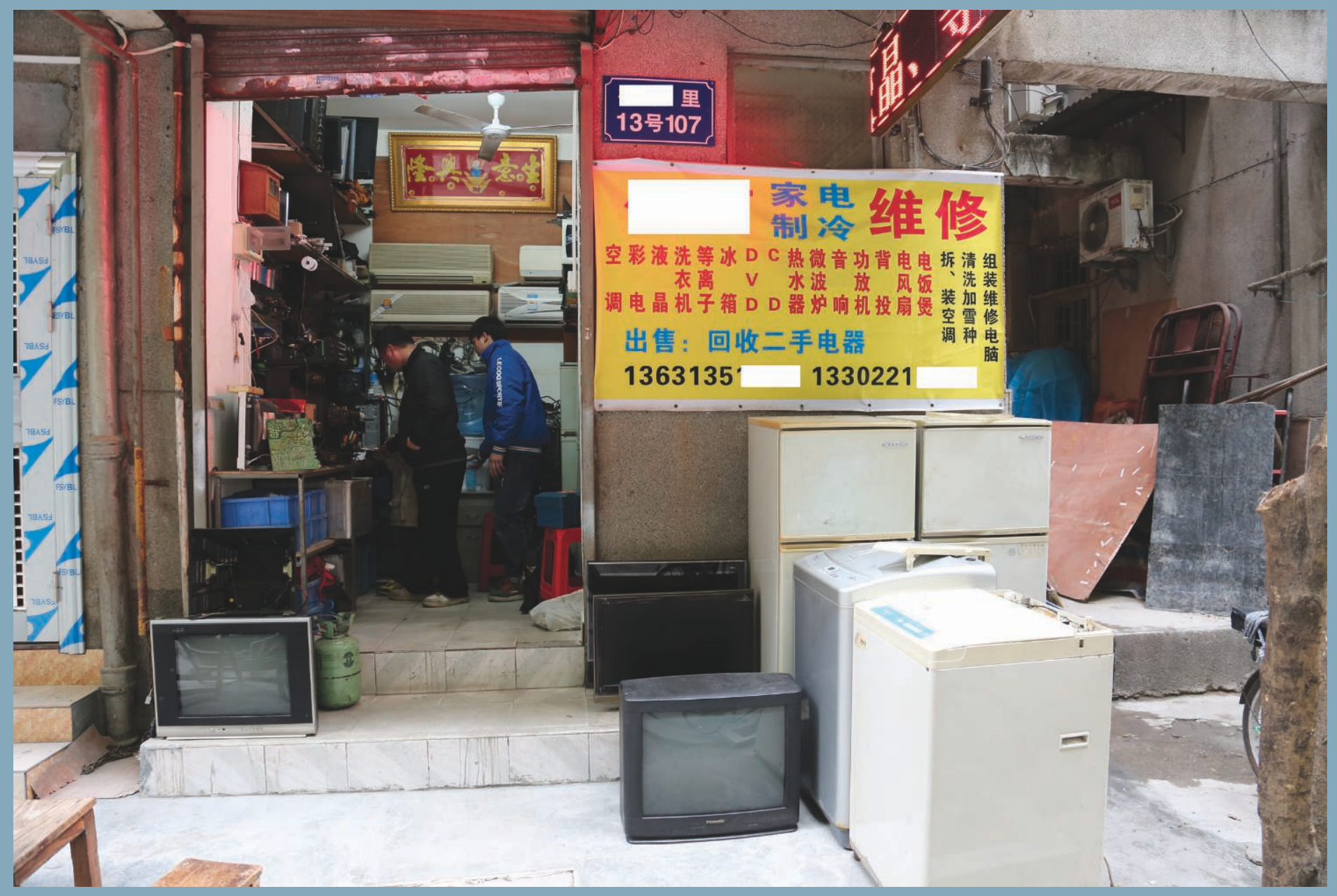




\section{"Fin de vie " et renaissance clandestine en Chine du Sud}

Quand des « déchets " redeviennent des écrans plats

Depuis plus d'une décennie, les appareils électroniques d'occasion passent pour des « déchets». On s'intéresse avant tout aux matériaux de valeur qui peuvent en être extraits, à la pollution qui résulte de leur élimination ou de leur recyclage imparfait ainsi qu'à l'injustice mondiale qui conduit les régions pauvres du globe à accueillir et à transformer les biens de consommation abandonnés dans les régions riches. Mais un téléviseur hors d'état est-il vraiment l'équivalent d'un tas de vieux journaux ou d'une bouteille en plastique vide? Qu'en est-il des éventuelles différences aux niveaux des matérialités et des potentiels de réutilisation respectifs de ces objets?

Cet article se penche sur un des nombreux réseaux mondiaux qui permettent à des objets déclarés «en fin de vie» de renaître. Sur la base d'un cas pratique centré sur les moniteurs à écran plat en Chine du Sud, j'observe que le secteur de la récupération, de la remise à neuf et de la revente d'appareils et composants électroniques souffre d'une grande invisibilité et je cherche à comprendre pourquoi.

La première section de l'article fait le récit d'un moment dans mon enquête de terrain et emmène le/la lecteur/-trice sur mes traces. Parti à la recherche de « déchets » électroniques, je m'attendais à découvrir des objets abandonnés, un environnement contaminé, des preuves du gaspillage qu'occasionne le système capitaliste industriel et des victimes ou des complices de ce dernier. Or, en pistant un type d'objet particulier, les moniteurs à écran plat, je m’aperçois qu'il existe des objets convoités, des cycles matériels vertueux, de nature à préserver l'environnement, et des réseaux commerciaux transnationaux qui tirent habilement leur épingle d'un jeu pourtant rendu difficile par les États et les entreprises multinationales.

Cette recherche n'a pas été sans difficulté, en particulier en matière d'accès au terrain. J'ai dû venir à bout du silence, parfois même déchiffrer les mensonges de certains de mes interlocuteurs et je n'ai pu construire de relation proche qu'avec une poignée de personnes. Mon identité de chercheur, trop proche de celle de journaliste, rendait les gens soupçonneux. Pour parvenir à mes fins, il m’a donc fallu faire preuve d'inventivité, notamment en me mettant dans la peau 


\section{Le village de Xiaohe, vu de loin \\ Le terrain vague se situe sur le territoire de la mu- nicipalité de Canton, tandis que les bâtiments marquent le début du village.}

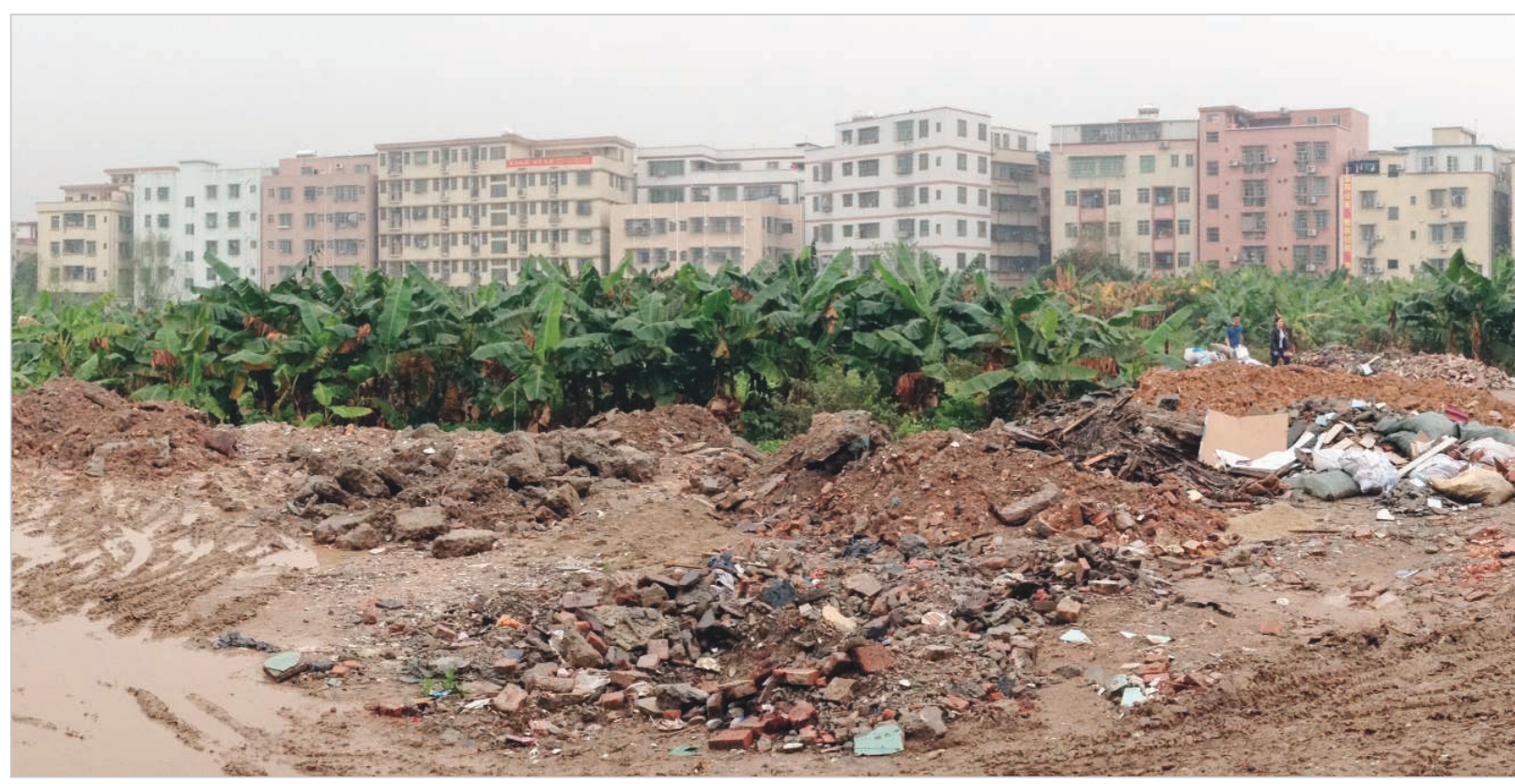

d'un négociant ou d'un consommateur. À la lecture, on aura, à raison, l'impression d'un monde en retrait, difficile à cerner, mais recelant moult surprises.

La seconde section de l'article reprend et complète le cas d'étude présenté dans la première. On y trouve une réflexion sur l'importance du secteur de la remise à neuf de matériel électronique et son rôle dans l'industrie au sens large. J'avance notamment que les difficultés rencontrées sur le terrain font partie de mon objet d'étude et qu'elles attestent son invisibilité. Ma recherche montre en effet que l'approche prédominante des appareils et composants électroniques usagés, qui consiste à leur attribuer automatiquement le statut de «déchets», conduit à mal interpréter leur matérialité, à ignorer des étapes importantes de leur parcours, à sous-estimer la puissance des mécanismes et des logiques économiques qui provoquent leur circulation, enfin, à condamner à la clandestinité les commerçants et les artisans qui œuvrent pour leur redonner vie.

On pourrait être tenté d'associer le secteur chinois de la réutilisation de matériel électronique au regain d'intérêt dont les pratiques de réparation bénéficient aujourd'hui en Europe et en Amérique du Nord. Je soutiens cependant qu'il fonctionne selon des logiques différentes. Tandis que la redécouverte de la réparation et de l'entretien doit beaucoup à une prise de conscience croissante, en particulier au sein des couches aisées de la population, des effets délétères des modes de consommation, de production et d'élimination contemporains, ainsi qu'à une recherche de solutions alternatives, la remise à neuf en Chine représente avant tout un gagne-pain pour les individus qui la pratiquent. Certes, ce type d'activités contribue à atténuer l'impact négatif du capitalisme industriel, ce qui mérite d'être mis en évidence et valorisé, mais elles dépendent des modes de fonctionnements fondamentaux de ce système et, par conséquent, ne les remettent pas en question. 


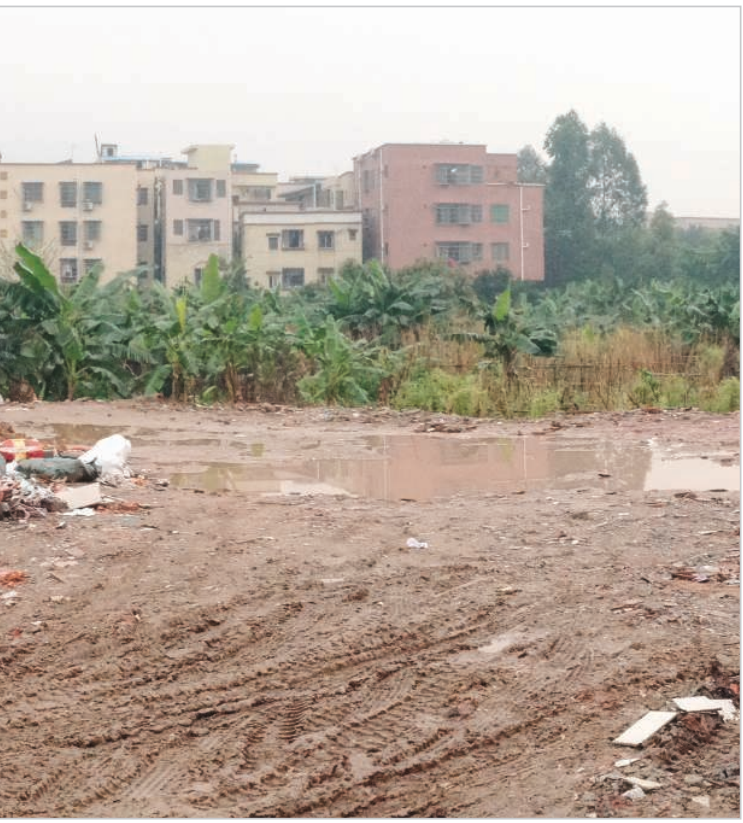

\section{En ligne}

Retrouvez l'article complet sur revues.org, TechniquesECulture 65-66 « Réparer le monde. Excès, reste et innovation » : http://tc.revues.org/

\section{I'auteur}

Yvan Schulz est doctorant à l'Institut d'ethnologie (université de Neuchâtel). Sa recherche porte sur le recyclage (au sens large) des appareils électriques et électroniques en Chine et a pour but d'expliquer l'évolution de cette industrie, notamment sous l'angle de la «formalisation» promue par le gouvernement central.

\section{Iconographie}

Image d'ouverture : Un atelier de réparation à Canton. (C) Yvan Schulz.

1. (C) Yvan Schulz.

\section{Pour citer cet article}

Schulz, Y. 2016 «"Fin de vie" et renaissance clandestine en Chine du Sud. Quand des "déchets" redeviennent des écrans plats », TechniquesE Culture 65-66 « Réparer le monde. Excès, reste et innovation », p. 158-161. 